\title{
THE RADIO LIGHT CURVE OF VA7I TAURI
}

\author{
J.-P. Caillault ${ }^{*}$, J. Patterson ${ }^{\dagger}$, and D. Skillman ${ }^{\S}$ \\ *Department of Pliysics and Astronomy, University of Georgia \\ Allens, GA 30607

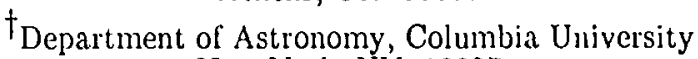 \\ New York, NY 10027

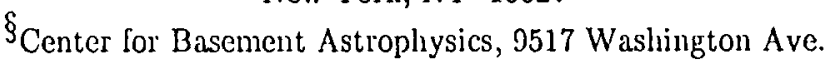 \\ Laurel, MD 20707
}

\begin{abstract}
We have observed the white dwarf - K (lwarf eclipsing binary system VA71 Tauri with the VLA ${ }^{1}$. We have detected in the radio flux an interesting dip centered near pliase zero (the plase of white dwarf eclipse) and a $6 \mathrm{mJy}$ flare shortly after mid-eclipse at phase 0.15 . The best possible explanation for the dip is the self-erlipse of a large radio-emitting cloud anchored to a particulart spot on the secondary, namely the sub-white dwarf point. The $6 \mathrm{~cm}$ Mare observation and sudden variations seen in II $\alpha$ suggest that this spot is an active flaring region.
\end{abstract}

\section{INTRODUCTION}

In the last 16 years one of the most extensively observed stars has been V171 Tauri $\left(=B D+10^{\circ} 516\right)$, the gth magnitude eclipsing binary in the Hyades. Nelson and Young (1970)/1/ discovered that the star shows eclipses of a small, hot object, with the mass and radius of a white dwarf. Young and Capps (1971) /2/ demonstrated from proper motion data that it is almost certainly a member of the Hyades, and spectroscopic studies /3,4/ established the basic system parameters: a $0.7 \mathrm{M}_{0} \mathrm{~K}$ dwarf and a $0.8 \mathrm{M}_{0}$ whitc dwarf, with an orbital inclination of $80^{\circ}$ and a period of 12.5 hours.

V471 Tau demonstrates many of the properties normally attributed to classical RS CVn stars /5/ and also exhibits flaring properties which characterize the BY Dra stars /6/. RS CVn-type billaries are probably the most common kind of radio-detected stars, with the possible exception of flare stars. Their radio emission is iniense, highly variable, and often circularly polarized /7/. VA71 Tau has been observed previously with the VLA, but only in a delection experiment; the observed quiescent flux was $\sim 0.8 \mathrm{mJy} / 8 /$.

The photometric coverage of V471 Tau /9,10/ permits us to know the binary phase at which the magnetic starspot is face on. Since the suggested mechanism for the quiescent emission of late-type stars observed by the VLA is thought to be magnetic in character, it is reasonable to expect V471 Tau to show similar modulation in the microwave region, unless the radio emission region greatly exceeds the star in sizc. In fact, the maximum microwave emission of the eclipsing binary system $Y Y$ Gem (dM1c $+\mathrm{dM} 1 \mathrm{c})$ was seen to occur in phase with the meridian passage of the large starspot group arid active region on the secondary star in this system $/ 11 /$.

Finally, there are two additional major reasons for determining the microwave properties of V471 Tau: 1.) its large rotational velocity $(84 \mathrm{~km} / \mathrm{sec})$ provides us with previously unattained access to the high-velocity regime for active $K$ dwarfs, and 2.) the $K$ dwarf will soon (in $~ 2 \times 10^{7}$ years) become the mass-losing secondary in a cataclysmic variable, having evolved from a "common envelope binary" phase of evolution, in which the $K$ dwarf was spiralling through the outer atmosphere of a red giant $/ 12 /$.

Hence, we have used the VLA to observe V471 Tau with the intention of using the high binary inclination to eclipse the region of radio emission, and thereby to learn its location and size.

1 - The VLA is a facility of the NRAO, which is operated by Associated Universities, Inc., under contract with the National Science Foundation. 


\section{OBSERVATIONS AND RESULTS}

We observed V471 Tauri for 8 consecutive hours on two separate days with the VLA in the C-D array. These $6 \mathrm{~cm}$ observations took place on 22-23 and 26-27 January 1987. The phase calibrator used was $0400+258 ; 3 \mathrm{C} 48$, with an assumed flux of $5.6 \mathrm{Jy}$, was used as the flux calibrator. The data were reduced in the standard manner, i.c., by making maps in each of the four Stokes' parameters I, U, V, and Q (no significant polarization was scen $[<10 \%])$. In order to obtain the $6 \mathrm{~cm}$ light curve discussed below, the reduction process was repeated for shorter sequential selected time segments.

The $6 \mathrm{~cm}$ light curve that we obtained is shown in Figure 1. On 22-23 January, the source was weak and slowly varying, but the $26-27$ January observation showed a brighter source with an interesting dip centered near phase zcro (the phase of white dwarf eclipse) and a $6 \mathrm{mJy}$ flare shortly after mid-eclipse at phase $\sim 0.15$; this llare then decayed back down to its preflare level $(\sim 2-3 \mathrm{mJy})$ in about 2 hrs.

Our uriarly simultancous optical light curve (Figure 2) obtained with an automated $0.32 \mathrm{~m}$ telescope /13/ in carly Jannary 1987, showed the optical wave ininimum to be near phase 0.5 . The superposed smooth double sinusoid arises from the tidal distortion in the Roche lobe, hence the light curve suggests a dark spot face-on near phatse 0.5 .

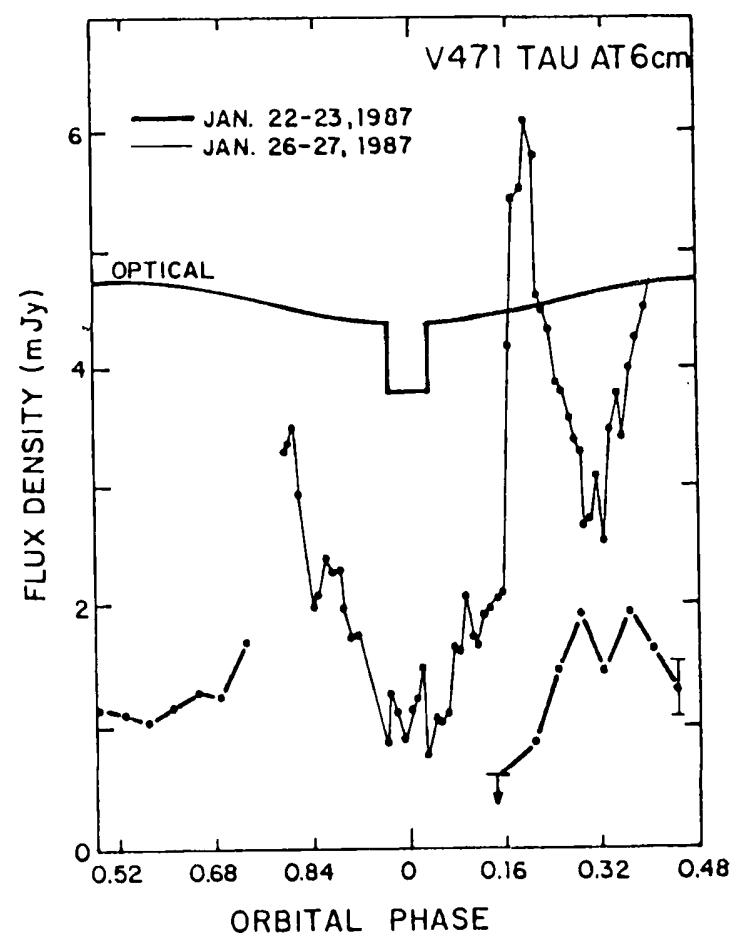

Fig. 1 The orbital light curve at $\lambda=6 \mathrm{~cm}$, with the nearly simultancous optical light curve superimposed, sans scaling. Note the dip in the radio light curve near phase zero.

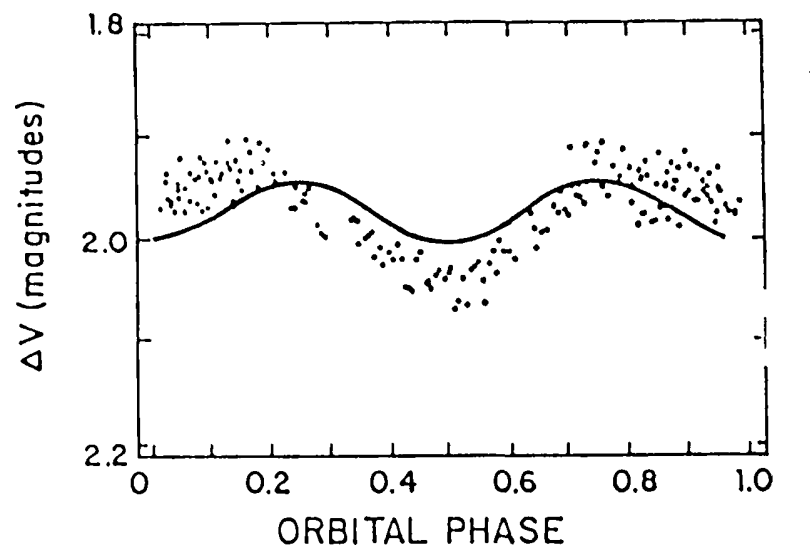

Fig. 2 Optical light curve of vit1 Tau, obtained in eariy January 198i. The superposed smooth double sinusoid arises from the idal distortion in the Ruche lobe, sc !he ligint curve suggests a dark spot face-on near phase 0.5 . 


\section{DISCUSSION}

We have considered three possible explanations for the dip: (1) the eclipse of a large radio-emitting cloud centered on the presumably magnetic white dwarf; (2) the self-eclipse of a large radio-cmitting cloud anchored to a particular spot on the secondary, namcly the sub-white dwarf point; and (3) random variability.

We think that (2) is the leading-jossibility, because of the optical light curve. If, as is widely believed, the optical minimum is a result of the presence of a dark starspot, then the "spot", ind any structures associated with it, woukl suffer a broad eclipse near phase zero - consistent with the radio light curve. The $6 \mathrm{~cm}$ flare observation and sudden variations scen in $\mathrm{H} \alpha / 14 /$ suggest that this spot is an active flaring region.

In order to exclude (3) we simply need more data, and to exclude (1) we should obtain data when the spot is not nearly face-on to the white dwarf (i.e., after it has drifted adequately in longitude). In order to guarantee that the latter does not occur, it is advisable to obtain two orbital light curves separated by $\sim 60-90$ days (to allow the spot to drift adequately in longitude).

Additional VLA time has been scheduled (separated by 150 days) in order to verify if this eclipse of radio emission is a reproducible feature of the light curve. The favorable geometry and the very promising first observation makes it rather likely that we wili realiy learn the size and location of the radio emission relative to the starspot - a crucial link that has not yet been securely established for any stellar radio source.

\section{REFERENCES}

1. Nelson, Fi. and Young, A., Publ.Astron.Soc.Pac. 82, 699 (1970).

2. Young, A. and Capps, P. W., Astrophys.J.(Letters) 166, L81 (1971).

3. Young, A. and Nelson, B., Astrophys.J. 173, 653 (1972).

4. Young, A. and Lanning, H., Publ.Astron.Soc.Pac. 87, 461 (1975).

5. Hall, D. S., in Multiple Periodic Variable Stars, ed. W.S. Fitch, (Dordrecht:Reidel), p. 287 (1976).

6. Bopp, B. W. and Fekel, F., Jr., Astron.J. 82, 490 (1977).

7. Mutel, R. L. and Lestrade, J. F., Astron.J. 90, 493 (1985).

8. Gibson, D., in Radio Stars, eds. R. IIjellming and D. Gibson, (Dordrecht:Reidel), p. 213 (1985).

9. Skillman, D. R. and Patterson, J., Astron.J. 96, 976 (1988).

10. Guinan, E. and Sion, E., Bull.Am.Astron.Soc. 13, 817 (1983).

11. Linsky,J. L. and Gary, D. E., Astrophys.J. 274, 776 (1983).

12. Paczynski, B., in IAU Symposium No. 73: Structure and Evolution of Close Binary Systems, eds. P. Eggleton, S. Mitton, and J. Whelan, (Dordrecht:Reidel), p. 75 (1976).

13. Skillınan, D. R., Sky and Telesc. 61, 71 (1981).

14. Young, A., Skumanich, A., and Paylor, V., Bull.Am.Astron.Soc. 18, 978 (1986). 